\title{
Single Atoms in an Optical Dipole Trap: Towards a Deterministic Source of Cold Atoms
}

\author{
D. Frese, B. Ueberholz, S. Kuhr, W. Alt, D. Schrader, V. Gomer, and D. Meschede \\ Institut für Angewandte Physik, Universität Bonn, Wegelerstrasse 8, D-53115 Bonn, Germany
}

(Received 9 March 2000)

\begin{abstract}
We describe a simple experimental technique which allows us to store a small and deterministic number of neutral atoms in an optical dipole trap. The desired atom number is prepared in a magneto-optical trap overlapped with a single focused Nd:YAG laser beam. Dipole trap loading efficiency of $100 \%$ and storage times of about one minute have been achieved. We have also prepared atoms in a certain hyperfine state and demonstrated the feasibility of a state-selective detection via resonance fluorescence at the level of a few neutral atoms. A spin relaxation time of the polarized sample of $4.2 \pm 0.7 \mathrm{~s}$ has been measured. Possible applications are briefly discussed.
\end{abstract}

PACS numbers: $32.80 . P \mathrm{j}, 42.50 . \mathrm{Vk}$

Neutral atoms can conveniently and at very low kinetic energy be stored in a magneto-optical trap (MOT) [1], not only in large quantities but also in small and exactly known numbers of up to 20 single atoms [2,3]. For several applications, for instance, in cavity quantum electrodynamics [4], it is of interest to use perfectly controlled or deterministic samples of atoms for further experiments involving quantum interactions of an exactly known number of atoms. Full control of all internal and external atomic degrees of freedom is necessary in such applications but cannot be achieved in a MOT since, due to its dissipative character, all degrees of freedom are intimately mixed. In order to overcome this problem one can therefore combine the operating convenience of the MOT for isolated atoms with the advantages for quantum manipulation offered by the nearly conservative potential of optical dipole traps. The interest in optical dipole traps [5] as an elegant and simple way to store laser-cooled neutral atoms has rapidly increased within the last few years [6]. Far-off-resonance optical dipole traps [7] can confine atoms in all ground states for a long time with a very small ground-state relaxation rate [8]. Subwavelength localization of cold atoms can be achieved in micropotentials of a 3D interference pattern (so-called optical lattices [9]). Such experiments always operate with at least several thousands of atoms; the atom number cannot be determined exactly and is controlled only on average.

In the present work we load a small and exactly known atom number into an optical dipole trap with $100 \%$ efficiency, opening up a route to a novel kind of cold atom sources free of the indeterminism intrinsic to usual sources like atomic beams. We have also demonstrated the feasibility of a state-selective detection at the level of a few neutral atoms and measured a long spin relaxation time of some seconds. Together with recently demonstrated Raman sideband cooling [10] and the generation of nonclassical motional states of atoms in standing-wave dipole traps [11], this system promises to be a new basis for future experiments with full control of all atomic degrees of freedom. One of the most interesting possibilities would be long-time localization of more than one atom within a mode of a high finesse cavity.

The relevant part of the apparatus is shown in Fig. 1; details have been described elsewhere $[3,12]$. The number of trapped atoms and its temporal fluctuations in the MOT are determined by the balance between loading from the atomic vapor and different loss mechanisms removing atoms from the trap. For experiments with few atoms a strong reduction of the capture rate from the gas phase is essential. In the first place, this can be achieved by reducing the partial atomic vapor pressure in the vacuum chamber. Stiff magnetic field gradients lead to an additional reduction of the capture rate [13]. The intratrap collision loss rate in our MOT with a gradient of $B^{\prime}=d B / d z=375 \mathrm{G} / \mathrm{cm}$ is comparable to the rate for background collisions [14]. Along with the base pressure below $10^{-10}$ mbar this governs the trapped atom number dynamics on a time scale of seconds (see Fig. 2). The average number of trapped atoms can easily be adjusted between 1 and 10 by changing the cesium vapor pressure.

We emphasize that a strong magnetic field gradient is not a necessary condition for trapping of small atom numbers, as has been demonstrated previously [2]. However, a small MOT volume tremendously improves the localization of trapped atoms. The spatial distribution of the fluorescence in our MOT measured by a CCD camera has a Gaussian distribution with $1 / e$ radius between 5 and $17 \mu \mathrm{m}$

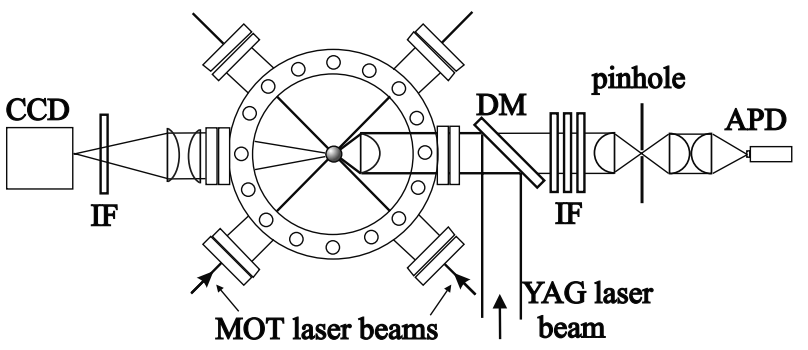

FIG. 1. Experimental setup. DM-dichroic mirror; IFinterference filters. In the image plane of the telescope a $150 \mu \mathrm{m}$ pinhole is placed for spatial filtering of the stray light. 


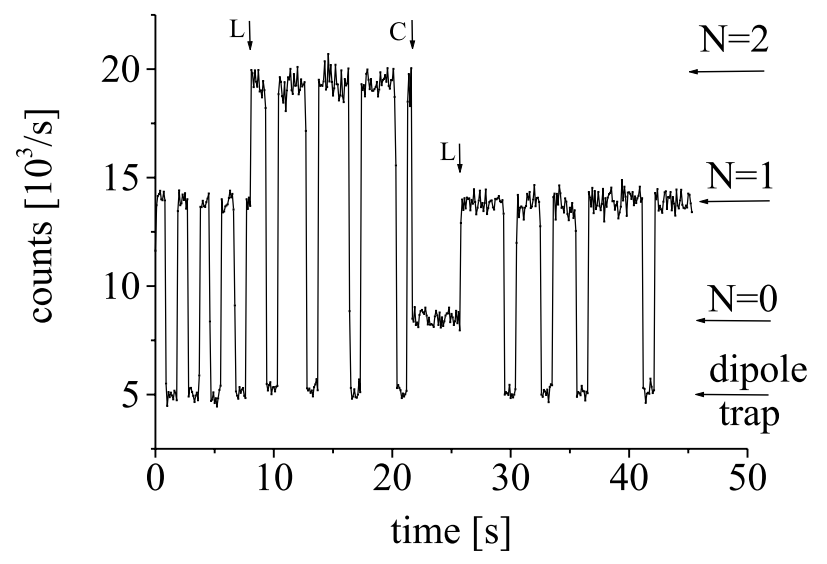

FIG. 2. Loading the dipole trap from the MOT with $100 \%$ efficiency. Shown are photon counts detected with the APD. Discrete signal levels correspond to an empty MOT $(N=0$, MOT stray light only), one and two atoms, respectively. Alternating to normal MOT operation the dipole trap is switched on and the MOT off for periods of $1 \mathrm{~s}$. The signal decreases to the lowest level which is due to the dipole trap laser stray light only. After that the atoms are recaptured into the MOT showing the same fluorescence level as before. During normal MOT operation atoms are occasionally loaded into the MOT from the background vapor $(\mathrm{L})$ or leave the MOT $(\mathrm{C}$, in this particular case as a result of a cold collision [14]).

depending on the laser intensity. The trap size was observed to be independent of the atom number $N$ up to $N=8$.

An atom strongly driven by resonant laser light emits an average fluorescence power of $\hbar \omega \Gamma / 2 \approx 3 \mathrm{pW}$, where $\Gamma$ is the natural linewidth, $\Gamma=2 \pi \times 5.2 \mathrm{MHz}$ for cesium. With a realistic overall detection efficiency of $10^{-3}$ it is necessary to discriminate about $3 \mathrm{fW}$ fluorescence power from a stray light background. Fluorescence of the atoms trapped in the MOT is observed with an avalanche photodiode (APD) in single photon counting mode with a measured photon detection efficiency of $50 \%$ at $\lambda=852 \mathrm{~nm}$. The fluorescence light is collected by a lens mounted inside the vacuum chamber and is sent through a telescope onto the APD; see Fig. 1. Typical photon counting rates are $(3-20) \times 10^{3} \mathrm{~s}^{-1}$ per atom depending on the detuning and intensity of the trapping laser. Well separated equidistant steps in the fluorescence signal allow us to monitor the number of trapped atoms in a noninvasive way and in real time [12]; see Fig. 2. During normal MOT operation we can thus easily choose a desired atom number to be transferred into the dipole trap.

The dipole trap consists of a single tightly focused $\mathrm{Nd}$ :YAG laser beam which is linearly polarized and superimposed on the MOT. We use the same lens inside the vacuum chamber both for focusing the dipole trap laser and for collecting the fluorescence. Because of the large difference between the wavelengths of the Nd:YAG laser $(1064 \mathrm{~nm})$ and the $\mathrm{D}_{2}$ line of cesium $(852 \mathrm{~nm})$, dipole trap laser radiation is easily blocked from the detection by interference filters. During simultaneous operation of both traps the fluorescence of the trapped atoms is sub- stantially reduced due to the light shift. Thus, the optimal geometrical overlap of the dipole laser with the MOT trapping volume can be achieved by minimizing the fluorescence. The dipole trap laser has a waist of about $5 \mu \mathrm{m}$ yielding a trap depth corresponding to $16 \mathrm{mK}$ and a maximum photon scattering rate of $190 \mathrm{~s}^{-1}$ at the center of the trap for a typical laser power of $2.5 \mathrm{~W}$.

Transfer of atoms between the two traps is accomplished through suitable timing sequences. First only the MOT is operated to collect atoms. To transfer these atoms into the dipole trap, the Nd:YAG laser is turned on a few ms before the MOT lasers are turned off. To recapture the atoms into the MOT, this procedure is reversed. By analyzing the resonance fluorescence we measure without any uncertainty the number of atoms right before transferring them into the dipole trap and directly after reloading the MOT; see Fig. 2. Note that the dipole trap provides a conservative potential and can therefore not capture atoms from the background vapor. The probability of capturing an atom by the MOT during the detection immediately after reloading the MOT is less than $1 \%$ and can be neglected.

Atoms can be caught by the dipole trap only at places where the dipole potential exceeds the atomic kinetic energy $E_{\mathrm{kin}}$, being of the order of $k_{B} T_{D}\left(T_{D}=\hbar \Gamma / 2 k_{B}=\right.$ $125 \mu \mathrm{K}$ for Cs) yielding the geometric loading efficiency $P=1-\left(E_{\mathrm{kin}} / U\right)^{w_{0}^{2} / r_{0}^{2}}$, where $U>E_{\mathrm{kin}}$ is the dipole potential in the trap center, $w_{0}$ and $r_{0}$ are $1 / e$ radii of the dipole trap and the MOT, respectively. Even for small MOT sizes used in our experiments (typically $\left.r_{0}=10 \mu \mathrm{m}\right) P$ is about $70 \%$. However, during a few $\mathrm{ms}$ of simultaneous operation of both traps, the MOT effectively cools the atoms down into the dipole potential. We have indeed found that $1 \mathrm{~s}$ after loading $N$ atoms from the MOT, the probability to find the same $N$ atoms in the dipole trap is more than $98 \%$ for all $N$ up to 7 . This is consistent with the measured dipole trap lifetime (see below) and $100 \%$ loading efficiency.

Varying the time spent by atoms in the dipole trap we have measured the fraction of atoms transferred back into the MOT and hence the lifetime in the dipole trap to be also independent of $N$. The results are shown in Fig. 3 demonstrating again $100 \%$ loading efficiency and a storage time of $51 \pm 3 \mathrm{~s}$. Each point shows an averaging over 400 atoms (about 100 single observations on $N$ atoms, $N$ varying from 1 to 7 ). The same procedure repeated with the dipole trap laser blocked is also presented in Fig. 3 as circle symbols. In this case atoms are stored in the quadrupole magnetic field as reported previously [3]. As expected, roughly half of the atoms are immediately lost after switching off the MOT lasers due to a statistical distribution of the spin orientations relative to the local magnetic field. From the fact that the atoms' lifetime in the magnetic trap is the same, we conclude that storage in both physically very different traps is limited by background gas pressure only.

Because of the large detuning of the dipole trap laser from atomic resonances the light shifts of both hyperfine 


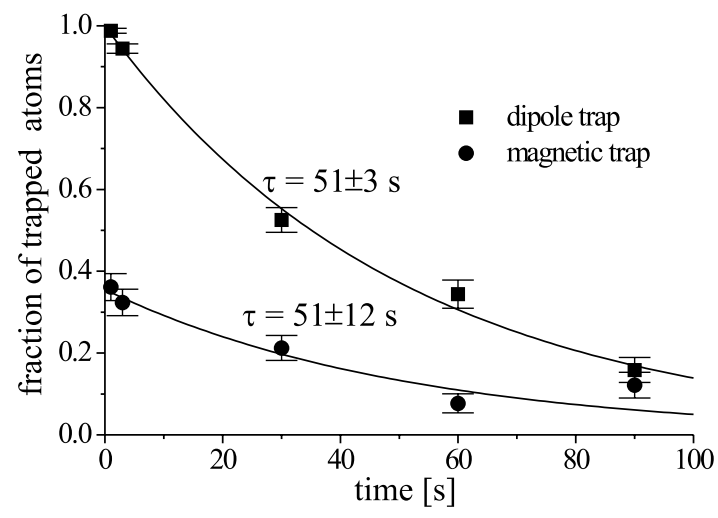

FIG. 3. Lifetime measurement for two types of traps. For details, see text.

ground states are nearly identical. We thus expect equal lifetimes in the dipole trap for atoms prepared in either hyperfine state. Still any initial preparation in a hyperfine state will be destroyed by off-resonant photon scattering in the dipole trap, yielding a relaxation of spin polarization. In the experiment, optical pumping is used to prepare an atom in a certain state. Introducing a delay of $8 \mathrm{~ms}$ between switching off the MOT repumping laser (resonant with the transition $F=3 \rightarrow F^{\prime}=4$ ) and the MOT cooling laser (in close resonance with the transition $F=4 \rightarrow F^{\prime}=5$ ) provides a convenient way to prepare the atoms in either the $F=3$ or $F=4$ state.

For state-selective detection the dipole trap laser is switched off by means of a Pockels cell $50 \mu \mathrm{s}$ before the detection laser is turned on. As a detection laser the MOT cooling laser is used, now tuned into exact resonance with the transition $F=4 \rightarrow F^{\prime}=5$, while the MOT repumping laser remains blocked. Atoms in the $F=4$ state contribute to the fluorescence signal, while atoms in the $F=3$ state stay dark.

A typical measurement sequence is shown in Fig. 4. After a storage of $100 \mathrm{~ms}$ in the dipole trap one clearly observes a fluorescence burst in the case that the $F=4$ state is prepared and probed whereas there is no additional signal from atoms initially prepared in the $F=3$ state. By varying the time between preparation of atoms in the dipole trap and probing we observed the relaxation of both hyperfine states towards an equilibrium due to spontaneous Raman scattering from the dipole trap laser, as given in Fig. 5. Each of these measurements was obtained by averaging over 90 atoms (30 runs with three trapped atoms each), following the scheme outlined above. The photon number in the detected fluorescence peak is proportional to both the population in the $F=4$ state and the stored atom number, the latter known exactly. The figure shows the population in the $F=4$ state extracted from the obtained data. Theoretical treatment reveals that the Raman scattering rate transferring population from one hyperfine state $F$ to the other $F^{\prime}$ is proportional to $2 F^{\prime}+1$. Spin-changing collisions can be neglected in this analysis [15].

The observed relaxation rate is almost 3 orders of magnitude smaller than the expected total photon scattering
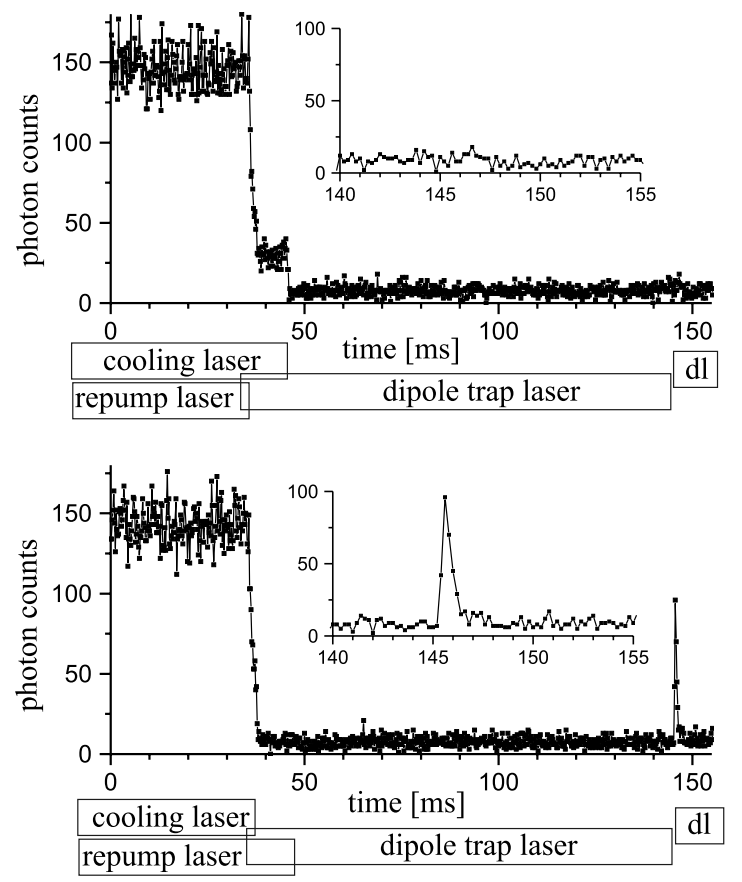

FIG. 4. State-selective detection of atoms in the $F=4$ state after $100 \mathrm{~ms}$ in the dipole trap. Integration time for one point is $200 \mu \mathrm{s}$. Frames below time axes show the switching sequence of all lasers used in the measurement and indicate their temporal overlaps; dl-detection laser. Top: Atoms are prepared in the $F=3$ state. Bottom: After preparation in the $F=4$ state. Inset: Enlarged view at the time the detection laser turned on.

rate at the trap center. The measured very long groundstate relaxation times of $4.2 \pm 0.7 \mathrm{~s}$ for $F=4$ and $3.3 \pm$ $0.6 \mathrm{~s}$ for $F=3$ clearly show a strong suppression of spontaneous Raman scattering processes. This effect is due to destructive interference of scattering amplitudes far from resonance and has recently been observed on optically trapped rubidium atoms and explained in [8]. For a Cs atom in a Nd:YAG dipole trap Raman scattering is suppressed compared to the Rayleigh scattering rate by a factor of 90 [16]. Although the experiment described here is sensitive only to the change of the hyperfine $F$ state,

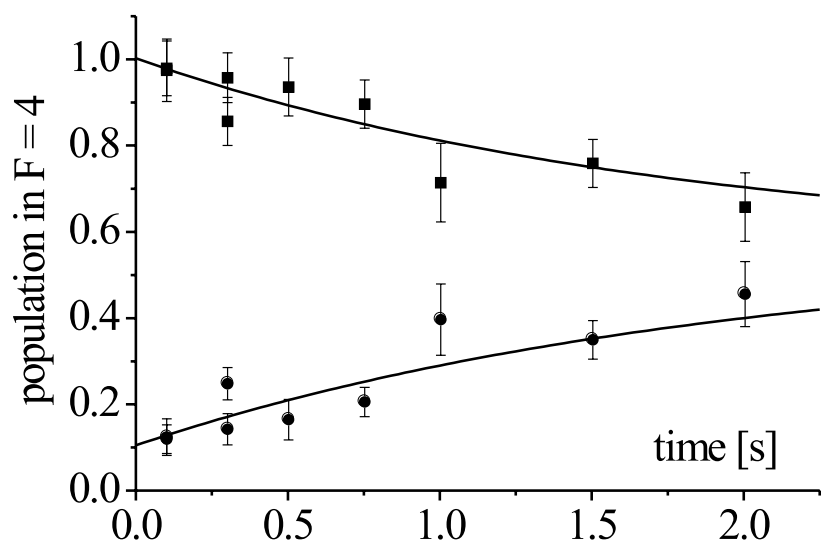

FIG. 5. Relaxation of hyperfine states due to Raman scattering. Shown is the measured population in the $F=4$ state after initial preparation in $F=4$ (squares) and in $F=3$ (circles). 
similarly long relaxation times are also expected for all Zeeman $m_{F}$ sublevels.

The remaining factor of about 8 in the ratio between the measured relaxation rate and the maximum total scattering rate can be explained by the oscillatory motion of the atoms in the dipole trap. The scattering rate is proportional to the time-averaged light intensity seen by the trapped atoms. In a Gaussian-shaped potential an average intensity 8 times smaller than the maximum intensity in the trap center corresponds to an oscillation amplitude of about $7 \mu \mathrm{m}\left(1.5 w_{0}\right)$. This estimation is supported by the following simple model of the loading process: During simultaneous operation of both traps the MOT cooling action is still at work for large distances from the trap center (a distance of $1.5 w_{0}$ corresponds to a light shift of $10 \Gamma$ in our case). Thus the MOT damps the atomic motion and effectively collects the atom into the dipole trap. Near the dipole trap center, however, the MOT light forces are too weak to enforce a further localization and after switching off the MOT we expect the corresponding oscillation amplitude of about $1.5 w_{0}$ in the conservative dipole potential.

As already mentioned, for state-selective detection we use the MOT cooling laser beams. As a result of the 3D character of the MOT light field the atom decays within about $400 \mu$ s into another hyperfine state thus terminating the fluorescence signal. Even in this situation we are able to detect on average three fluorescence photons per atom on a stray light background of 0.5 photons. There are natural ways to improve the detection scheme by using closed transitions in an optically pumped atom similar to single trapped ion experiments [17] and setting up different laser beam geometries. This should result in an improvement of the signal to noise ratio enabling us to detect the state of a single trapped atom with high certainty.

In summary, we have demonstrated $100 \%$ transfer efficiency of atoms from the MOT into the dipole trap. For times short compared to both the dipole trap storage time and the spin relaxation time, the experiment also shows the potential of the apparatus to prescribe the state of an exactly determined number of atoms. It is now conceivable to tailor dipole trapping laser fields for transporting a desired number of atoms to another location (optical tweezer for single atoms), e.g., into a resonator of high finesse. Together with state-selective detection this will open up a wide range of applications not possible so far with either single trapped ions or many neutral atoms in optical dipole traps.

Usual sources of neutral atoms like atomic beams or atoms released from a magneto-optical trap provide a flux of uncorrelated atoms. For some specific experiments, however, there is great interest in a source providing an arrival of a certain small number of atoms at time moments set by the experimentalist, i.e., with a $\delta$-like arrival probability distribution.

A promising application is experiments on cavity QED and quantum information processing. Quantum logic gates can be implemented by entangling neutral atoms through the exchange of cavity photons. The feasibility of using this atom-cavity interaction in the optical range has already been demonstrated by the groups of Kimble [18] and Rempe [19]. In particular, they have trapped a single atom inside a cavity. However, in these experiments the atoms enter the cavity in a random way after being released from a MOT and it is impossible to have a certain small number of atoms in the cavity on demand. As noted in [20] simultaneous strong coupling of more than one atom to the cavity can minimize the effects of decoherence (cavity decay) in a quantum gate implementation. This would produce an entanglement between internal states of two atoms - a basic element for quantum information processing.

[1] E. L. Raab et al., Phys. Rev. Lett. 59, 2631 (1987).

[2] Z. Hu and H. J. Kimble, Opt. Lett. 19, 1888 (1994); F. Ruschewitz et al., Europhys. Lett. 34, 651 (1996); P. A. Willems et al., Phys. Rev. Lett. 78, 1660 (1997).

[3] D. Haubrich et al., Europhys. Lett. 34, 663 (1996).

[4] Cavity Quantum Electrodynamics, edited by P. Berman (Academic Press, San Diego, 1994).

[5] J. P. Gordon and A. Ashkin, Phys. Rev. A 21, 1606 (1980); S. Chu et al., Phys. Rev. Lett. 57, 314 (1986).

[6] For a recent review, see R. Grimm, M. Weidemüller, and Y. B. Ovchinnikov, Adv. At. Mol. Opt. Phys. 42, 95 (2000).

[7] S. Rolston et al., Proc. Soc. Photo-Opt. Instrum. Eng. 1726, 205 (1992); J. D. Miller, R. A. Cline, and D. J. Heinzen, Phys. Rev. A 47, R4567 (1993).

[8] R. A. Cline et al., Opt. Lett. 19, 207 (1994).

[9] P. S. Jessen and I. H. Deutsch, Adv. At. Mol. Opt. Phys. 36, 91 (1996), and references therein.

[10] S. Hamman et al., Phys. Rev. Lett. 80, 4149 (1998); H. Perrin et al., Europhys. Lett. 42, 395 (1998).

[11] I. Bouchoule et al., Phys. Rev. A 59, R8 (1999); M. Morinaga et al., Phys. Rev. Lett. 83, 4037 (1999).

[12] V. Gomer et al., Phys. Rev. A 58, R1657 (1998); V. Gomer et al., Appl. Phys. B 67, 689 (1998).

[13] A. Höpe et al., Europhys. Lett. 22, 669 (1993); D. Haubrich et al., Opt. Commun. 102, 225 (1993).

[14] B. Ueberholz et al., J. Phys. B 33, L135 (2000).

[15] Spin-changing collisions release a kinetic energy of at least $0.2 \mathrm{~K}$ per atom for Cs which is much larger than the dipole trap depth and lead to immediate loss of trapped atoms. Since the measured lifetime is independent of the atom number, the collisional rate in the dipole trap is smaller than $0.02 \mathrm{~s}^{-1}$.

[16] Homogeneous distribution over $m_{F}$ sublevels assumed.

[17] W. M. Itano et al., Phys. Rev. A 47, 3554 (1993).

[18] C. J. Hood et al., Phys. Rev. Lett. 80, 4157 (1998); J. Ye, D. W. Vernooy, and H. J. Kimble, Phys. Rev. Lett. 83, 4987 (1999); C. J. Hood et al., Science 287, 1447 (2000).

[19] P. Münstermann et al., Opt. Commun. 159, 63 (1999); P. Pinkse et al., Nature (London) 404, 365 (2000); P. Münstermann et al., Phys. Rev. Lett. 84, 4068 (2000).

[20] T. Pellizzari et al., Phys. Rev. Lett. 75, 3788 (1995). 